\title{
A Ten-Qubit Solid-State Spin Register with Quantum Memory up to One Minute
}

\author{
C. E. Bradley, ${ }^{1,2,}$ J. Randall, ${ }^{1,2, \dagger}$ M. H. Abobeih, ${ }^{1,2}$ R. C. Berrevoets, ${ }^{1,2}$ M. J. Degen, ${ }^{1,2}$ \\ M. A. Bakker, ${ }^{1,2}$ M. Markham, ${ }^{3}$ D. J. Twitchen, ${ }^{3}$ and T. H. Taminiau ${ }^{1,2, *}$ \\ ${ }^{1}$ QuTech, Delft University of Technology, P.O. Box 5046, 2600 GA Delft, Netherlands \\ ${ }^{2}$ Kavli Institute of Nanoscience Delft, Delft University of Technology, \\ P.O. Box 5046, 2600 GA Delft, Netherlands \\ ${ }^{3}$ Element Six, Fermi Avenue, Harwell Oxford, Didcot, Oxfordshire, OX11 0QR, United Kingdom
}

(Received 9 May 2019; published 11 September 2019)

\begin{abstract}
Spins associated with single defects in solids provide promising qubits for quantum-information processing and quantum networks. Recent experiments have demonstrated long coherence times, highfidelity operations, and long-range entanglement. However, control has so far been limited to a few qubits, with entangled states of three spins demonstrated. Realizing larger multiqubit registers is challenging due to the need for quantum gates that avoid cross talk and protect the coherence of the complete register. In this paper, we present novel decoherence-protected gates that combine dynamical decoupling of an electron spin with selective phase-controlled driving of nuclear spins. We use these gates to realize a ten-qubit quantum register consisting of the electron spin of a nitrogen-vacancy center and nine nuclear spins in diamond. We show that the register is fully connected by generating entanglement between all 45 possible qubit pairs and realize genuine multipartite entangled states with up to seven qubits. Finally, we investigate the register as a multiqubit memory. We demonstrate the protection of an arbitrary single-qubit state for over $75 \mathrm{~s}$ - the longest reported for a single solid-state qubit - and show that two-qubit entanglement can be preserved for over $10 \mathrm{~s}$. Our results enable the control of large quantum registers with long coherence times and therefore open the door to advanced quantum algorithms and quantum networks with solid-state spin qubits.
\end{abstract}

DOI: 10.1103/PhysRevX.9.031045

Subject Areas: Condensed Matter Physics,

Quantum Physics,

Quantum Information

\section{INTRODUCTION}

Electron and nuclear spins associated with single defects in solids provide a promising platform for quantum networks and quantum computations [1,2]. In these hybrid registers, different types of spins fulfill different roles. Electron spins offer fast control [3-13] and high-fidelity readout $[7,14,15]$ and can be used to control and connect nuclear spins [15-21]. Furthermore, electron-electron couplings enable on-chip connectivity between defects [19, 22,23], while coupling to photons [12,24-28] allows for the realization of long-range entanglement links [29-31]. Nuclear spins provide additional qubits with long coherence

\footnotetext{
T.H.Taminiau@TUDelft.nl

${ }^{\dagger}$ These authors contributed equally this work.
}

Published by the American Physical Society under the terms of the Creative Commons Attribution 4.0 International license. Further distribution of this work must maintain attribution to the author(s) and the published article's title, journal citation, and DOI. times that can be used to store and process quantum states [16,17,21,24,32-35].

Recent experiments have demonstrated various schemes for high-fidelity two-qubit gates [34,36-41], as well as basic quantum algorithms [36,42] and error-correction codes $[16,17]$. However, to date, these systems have been restricted to few-qubit registers: The largest reported entangled state contains three qubits $[16,17,43]$. Larger quantum registers are desired for investigating advanced algorithms and quantum networks [44-46]. Such multiqubit registers are challenging to realize due to the required gates that selectively control the qubits and at the same time decouple unwanted interactions in order to protect coherence in the complete register.

In this work, we develop a novel gate scheme based upon selective phase-controlled driving of nuclear spins interleaved with decoupling sequences on an electron spin. These gates enable high-fidelity control of hitherto inaccessible nuclear spin qubits. We combine these gates with previously developed control techniques $[14,16,47]$ to realize a ten-qubit register composed of a diamond nitrogen-vacancy (NV) center, its ${ }^{14} \mathrm{~N}$ nuclear spin, and eight ${ }^{13} \mathrm{C}$ 


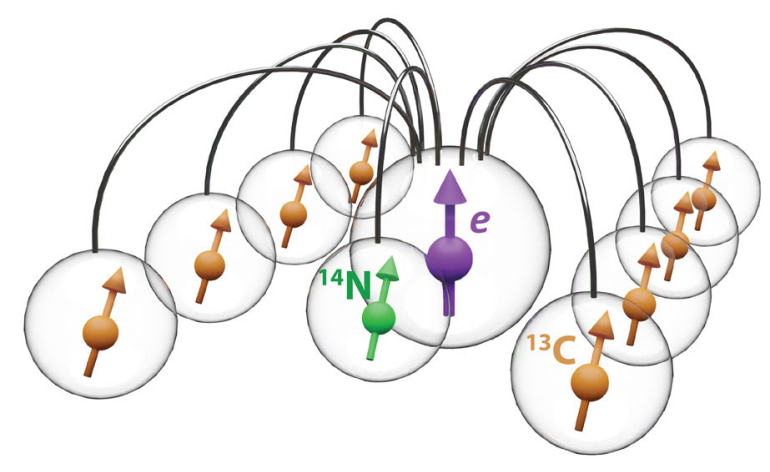

FIG. 1. Illustration of the ten-qubit register developed in this work. The electron spin of a single NV center in diamond acts as a central qubit and is connected by two-qubit gates to the intrinsic ${ }^{14} \mathrm{~N}$ nuclear spin and a further eight ${ }^{13} \mathrm{C}$ nuclear spins surrounding the NV center.

spins (Fig. 1). We show that the register is fully connected by preparing entangled states for all possible pairs of qubits. Furthermore, by also decoupling nuclear-nuclear interactions through echo sequences, we generate $N$-qubit Greenberger-Horne-Zeilinger (GHZ) states and witness genuine multipartite entanglement for up to seven spins. Finally, we investigate the coherence properties of the register. We measure coherence times up to 63(2) s and show that an arbitrary single-qubit state can be protected for over 75 s. Furthermore, we demonstrate that two-qubit entanglement can be preserved for over $10 \mathrm{~s}$.

\section{TWO-QUBIT GATES: THEORY}

We consider a NV center in diamond and surrounding ${ }^{13} \mathrm{C}$ nuclear spins. To realize a multiqubit register, we design single-qubit gates and electron-nuclear two-qubit gates to control the $\mathrm{NV}{ }^{14} \mathrm{~N}$ spin and several individual ${ }^{13} \mathrm{C}$ spins. The key challenges in these hybrid systems of multiple coupled spins are to maintain coherence on the electron-spin qubit and to avoid unwanted cross talk. In particular, the electron spin continuously couples to all ${ }^{13} \mathrm{C}$ spins through the hyperfine interaction, and the dynamics of the electron spin and nuclear spins typically occur on very different timescales [36]. To address these issues, a variety of decoherence-protected gates in which decoupling sequences on the electron spin are combined with nuclear spin control have been investigated [36,37,39,40,48-52]. Here we develop and demonstrate a novel electron-nuclear two-qubit gate based upon phase-controlled radiofrequency (rf) driving of nuclear spins interleaved with dynamical decoupling (DD) of the electron spin. We refer to this scheme as a DDrf gate. Our scheme enables the control of additional ${ }^{13} \mathrm{C}$ spins while offering improved flexibility in dynamical decoupling to optimize the electron-spin coherence and avoid unwanted cross talk.

To design a selective two-qubit gate, we utilize the hyperfine interaction which couples each nuclear spin to the electron spin. As this interaction depends on the relative position of the spin to the NV, different nuclear spins can be distinguished by their precession frequencies [48-50]. In the interaction picture with respect to the electron energy splitting, and neglecting nonsecular terms, the Hamiltonian describing the electron and a single ${ }^{13} \mathrm{C}$ nuclear spin is given by [48-50]

$$
H=\omega_{L} I_{z}+A_{\|} S_{z} I_{z}+A_{\perp} S_{z} I_{x},
$$

where $\omega_{L}=\gamma B_{z}$ is the nuclear Larmor frequency set by the external magnetic field $B_{z}$ along the NV axis, $\gamma$ is the ${ }^{13} \mathrm{C}$ gyromagnetic ratio, $S_{\alpha}$ and $I_{\alpha}$ are the spin- 1 and spin- $1 / 2$ operators of the electron and nuclear spins, respectively, and $A_{\|}$and $A_{\perp}$ are the parallel and perpendicular hyperfine components, respectively.

To control the nuclear spin, we apply rf pulses of Rabi frequency $\Omega$, phase $\phi$, and frequency $\omega$. To target a specific nuclear spin, we set $\omega=\omega_{1}$, where $\omega_{1}=$ $\sqrt{\left(\omega_{L}-A_{\|}\right)^{2}+A_{\perp}^{2}}$ is the nuclear spin-precession frequency when the electron is in the $m_{s}=-1$ spin projection. In the following, we assume $\left(\omega_{L}-\omega_{1}\right) \gg \Omega$, such that driving of the nuclear spin is negligible while the electron is in the $m_{s}=0$ spin projection, and set $A_{\perp}=0$ for simplicity (see the Supplemental Material [53] for the general case). Considering only the $m_{s}=\{0,-1\}$ subspace, with the addition of $\mathrm{rf}$ driving and in a rotating frame at the rf frequency, the Hamiltonian of Eq. (1) becomes $[36,53]$

$$
\begin{aligned}
H= & |0\rangle\langle 0| \otimes\left(\omega_{L}-\omega_{1}\right) I_{z} \\
& +|1\rangle\langle 1| \otimes \Omega\left[\cos (\phi) I_{x}+\sin (\phi) I_{y}\right],
\end{aligned}
$$

where $|0\rangle(|1\rangle)$ indicates the electron $m_{s}=0\left(m_{s}=-1\right)$ spin projection. In this picture, for the electron in state $|0\rangle$, the nuclear spin undergoes precession around the $\hat{z}$ axis at frequency $\left(\omega_{L}-\omega_{1}\right)=A_{\|}$. Conversely, while the electron is in the state $|1\rangle$, the nuclear spin is driven around a rotation axis in the $\hat{x}-\hat{y}$ plane defined by the phase of the rf field $\phi$.

To simultaneously decouple the electron spin from the environment, we interleave the rf pulses in a sequence of the form $(\tau-\pi-2 \tau-\pi-\tau)^{N / 2}$, where $\pi$ is a $\pi$ pulse on the electron spin, $2 \tau$ is the interpulse delay, and $N$ is the total number of electron decoupling pulses [Fig. 2(a)] [48-50]. We consider the evolution of the nuclear spin during this sequence separately for the two initial electron eigenstates: $|0\rangle$ and $|1\rangle$ [48-50]. We label each successive rf pulse by integer $k=1, \ldots, K$, where $K=N+1$ is the total number of rf pulses. If the initial electron-spin state is $|0\rangle$, only the even- $k$ rf pulses will be resonant and drive the nuclear spin [Fig. 2(b)]. Conversely, for initial state $|1\rangle$, the odd- $k$ pulses are resonant. The desired nuclear spin evolution can now be created by setting the phases $\phi_{k}$ of the rf pulses.

We construct both an unconditional rotation (singlequbit gate) and a conditional rotation (two-qubit gate). 


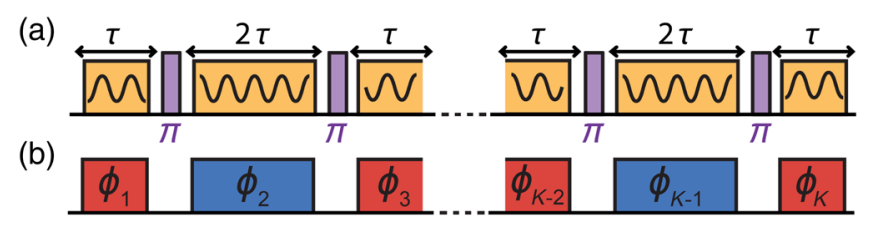

(c)
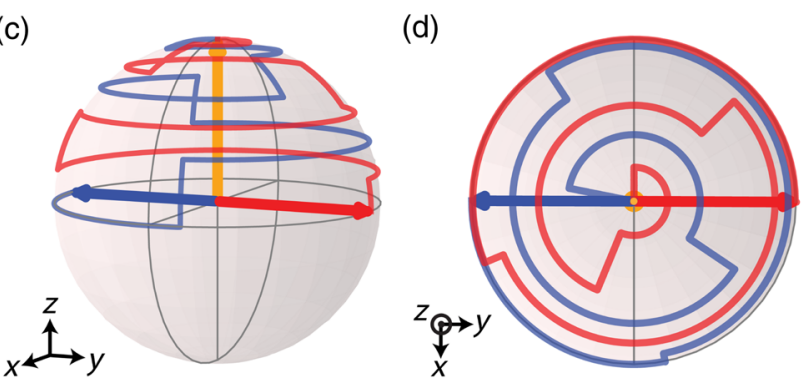

FIG. 2. (a) Illustration of the pulse sequence employed to realize a DDrf gate. Dynamical decoupling pulses on the electron spin (purple) are interleaved with rf pulses (yellow), which selectively drive a single nuclear spin. (b) Illustration showing that the initial state of the electron spin determines which rf pulses are resonant with the nuclear spin. If the electron spin starts in $|1\rangle$ $\left(m_{s}=-1\right)$, the odd rf pulses (red) are resonant. For initial electron state $|0\rangle\left(m_{s}=0\right)$, the even (blue) rf pulses are resonant. The phase of each rf pulse is adapted to create the desired nuclear spin evolution, accounting for periods of free precession according to Eq. (3). (c) Nuclear spin trajectory on the Bloch sphere for a conditional rotation with $N=8$ electron decoupling pulses. Starting from the initial nuclear state $|\uparrow\rangle$ (yellow), the red (blue) path shows the nuclear spin evolution for the case where the electron starts in the state $|1\rangle(|0\rangle)$. The final state vectors are antiparallel along the equator; therefore, the gate is a maximally entangling two-qubit gate. (d) Top-down view of (c).

To ensure that the sequential rf rotations build up constructively, the phases of each rf pulse should be set to account for the periods of nuclear spin precession between them, which build up in integer multiples of $\phi_{\tau}=\left(\omega_{L}-\right.$ $\left.\omega_{1}\right) \tau$. For the case where the electron starts in the state $|0\rangle$ (even $k$ ), the required sequence of phases is $\phi_{\tau}, 3 \phi_{\tau}, 5 \phi_{\tau}, \ldots$, while for the case where the electron starts in the state $|1\rangle$ (odd $k$ ), we require the sequence $0,2 \phi_{\tau}, 4 \phi_{\tau}, \ldots$ The required phases are therefore given by [53]

$$
\phi_{k}^{\prime}= \begin{cases}(k-1) \phi_{\tau}+\pi, & k \text { odd }, \\ (k-1) \phi_{\tau}, & k \text { even, }\end{cases}
$$

where the (optional) $\pi$ phase shift for the odd- $k$ sequence converts the unconditional rotation into a conditional rotation. By adding a further phase $\varphi$ to all pulses, we can also set the rotation axis of the gate. The rf pulse phases are thus summarized by $\phi_{k}=\varphi+\phi_{k}^{\prime}$.

With this choice of phases, the total evolution of the two-qubit system is given by $V=V_{z} V_{\text {CROT }}$. Here, $V_{z}$ is an unconditional rotation of the nuclear spin around $z$ [53], and $V_{\text {CROT }}$ is a conditional rotation of the nuclear spin depending on the electron state, given by

$$
\begin{aligned}
V_{\mathrm{CROT}}= & |0\rangle\langle 0| \otimes R_{\varphi}(N \Omega \tau) \\
& +|1\rangle\langle 1| \otimes R_{\varphi}(-N \Omega \tau),
\end{aligned}
$$

where $R_{\varphi}(\theta)=e^{-i \theta\left[\cos (\varphi) I_{x}+\sin (\varphi) I_{y}\right] / \hbar} . V_{\mathrm{CROT}}$ describes a controlled rotation of the nuclear spin with tuneable rotation angle (set by $N, \Omega$, and $\tau$ ) and rotation axis (set by $\varphi$ ). Setting $N \Omega \tau=\pi / 2$, a maximally entangling twoqubit operation is achieved, equivalent to a controlled-NOT gate up to local rotations. Example dynamics for a nuclear spin evolving under such a sequence are shown in Figs. 2(c) and 2(d).

Our design has several advantages. First, the gate allows nuclear spins with small or negligible $A_{\perp}$ to be controlled, thereby increasing the number of accessible nuclear spin qubits. Second, because the targeted dynamics are achieved by setting the rf phases and amplitudes, the interpulse delay $\tau$ of the decoupling sequence can be freely optimized to protect the electron coherence. This is in contrast to the gates described in van der Sar et al. [36], for which $\tau$ is restricted to a specific resonance condition for each spin, making multiqubit control challenging. Third, because our method does not rely on an average frequency shift over the two electron-spin states [37], our gates can also be used for selective control of nuclear spins coupled to spin-1/2 defects (such as the negatively charged group-IV color centers $[7,9,13,20,28,54,55])$ and via a contact hyperfine coupling, such as for donor spins in silicon [34] and SiMOS quantum dots [21]. Finally, because control is achieved through the rf field, a multitude of avenues for future investigation are opened up, such as parallelizing gates by frequency multiplexing and using shaped and composite pulses to mitigate dephasing and cross talk $[38,56,57]$.

\section{TWO-QUBIT GATES: EXPERIMENT}

Our experiments are performed at $3.7 \mathrm{~K}$ using a single NV center in diamond with natural abundance of carbon isotopes $\left(1.1 \%{ }^{13} \mathrm{C}\right)$. Further details of the sample and experimental setup can be found in the Supplemental Material [53]. As a starting point, we use the DDrf gate to identify and characterize ${ }^{13} \mathrm{C}$ nuclear spin qubits surrounding the NV center. If the electron spin is prepared in a superposition state, and the rf frequency is resonant with a nuclear spin in the environment, the entangling interaction [Eq. (4)] decoheres the electron spin. Therefore, varying the rf frequency $(\omega)$ performs spectroscopy of the nuclear spin environment. Figure 3 shows that multiple dips in the electron coherence can be observed, indicating selective interactions with several individual nuclear spins. Importantly, like other rfbased approaches [39,52], the DDrf sequence is sensitive to nuclear spins with small or negligible $A_{\perp}$. In addition to extending the number of qubits that can be controlled with a single NV center, this capability also enables the detection of additional spins when using the NV as a quantum sensor, 


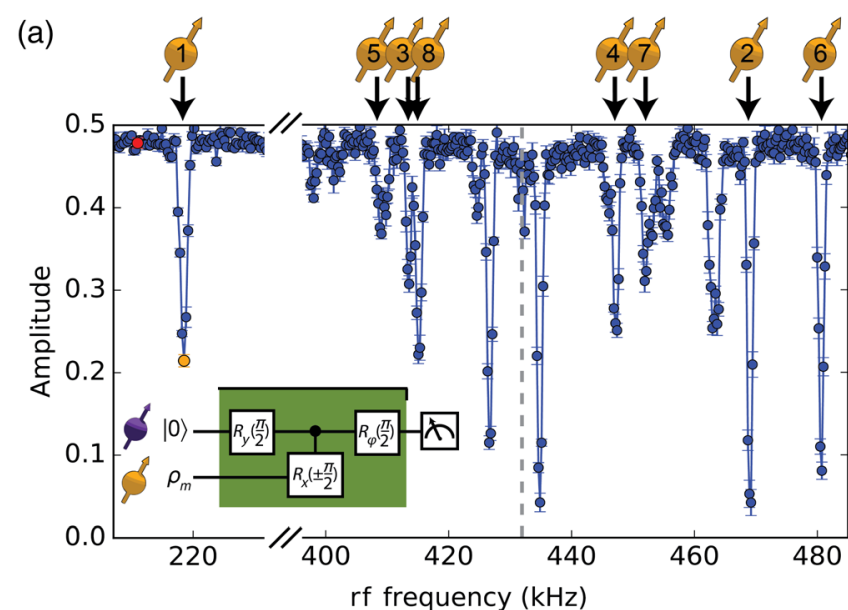

(b)
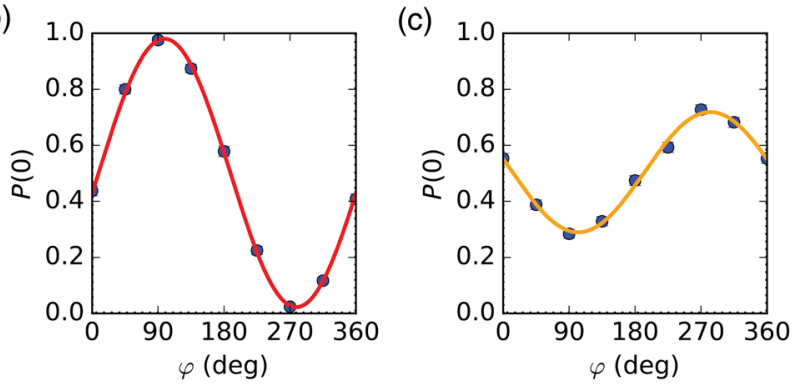

FIG. 3. (a) Nuclear spin spectroscopy. After preparing the electron in a superposition state, the DDrf gate [controlled $\pm \pi / 2$ rotation; see Eq. (4)] is applied for different $r f$ frequencies $\omega$. The electron spin is then measured along a basis in the equatorial plane defined by angle $\varphi$ (see inset). Each data point in (a) corresponds to the fitted amplitude $A$ of the function $f(\varphi)=$ $a+A \cos \left(\varphi+\varphi_{0}\right)$, where $\varphi$ is swept from 0 to $360 \mathrm{deg}$, and $\varphi_{0}$ accounts for deterministic phase shifts induced on the electron by the rf field. By fitting the amplitude, we distinguish such deterministic phase shifts from loss of coherence due to entangling interactions. The signals due to interaction with the eight ${ }^{13} \mathrm{C}$ spins used in this work are labeled. The dashed gray line indicates the ${ }^{13} \mathrm{C}$ Larmor frequency $\omega_{L}$. A detailed analysis of the spectrum is given in the Supplemental Material [53]. (b),(c) Example phase sweeps for two data points highlighted in red (b) and orange (c) in (a). Solid lines are fits to $f(\varphi)$. The DDrf gate parameters are $N=48$ and $\tau=8 \tau_{L}$, where $\tau_{L}=2 \pi / \omega_{L}(\approx 2.3 \mu \mathrm{s})$.

which we exploit in parallel work to realize 3D imaging of large spin clusters [58].

To verify the control offered by the DDrf two-qubit gate, we first demonstrate high-fidelity ancilla-based initialization and readout by preparation and tomography of a maximally entangled electron-nuclear state. To test the gate, we select a ${ }^{13} \mathrm{C}$ spin (spin $\mathrm{C} 1$, Fig. 3) with a strong parallel hyperfine component of $A_{\|} / 2 \pi=213.154(1) \mathrm{kHz}$ but a weak perpendicular hyperfine component $A_{\perp} / 2 \pi=$ $3.0(4) \mathrm{kHz}$ [53]. We exploit the freedom in choosing the interpulse delay by setting $\tau$ to an integer multiple of the ${ }^{13} \mathrm{C}$ Larmor period $\tau_{L}=2 \pi / \omega_{L}$, so that unwanted interactions between the electron spin and other ${ }^{13} \mathrm{C}$ spins in the environment are effectively decoupled $[47,59]$. The choice of Rabi frequency $\Omega$ is a trade-off between obtaining faster gate speeds, maintaining frequency selectivity, and minimizing additional noise from the electronic hardware [53].

The sequence to perform the state preparation and tomography experiment is shown in Fig. 4(a) [16,37]. We first initialize the electron spin in the state $|0\rangle$ by resonant optical excitation [14]. We then swap the state of the electron spin onto the ${ }^{13} \mathrm{C}$ spin and reset the electron spin. Next, we prepare the electron in a superposition state before performing the DDrf controlled-rotation gate, ideally preparing the electron-nuclear Bell state $\left|\psi_{\text {Bell }}\right\rangle=(|0+\rangle+|1-\rangle) / \sqrt{2}$, where $| \pm\rangle=(|\downarrow\rangle \pm|\uparrow\rangle) / \sqrt{2}$.

To perform quantum-state tomography on the two-qubit state, we first measure the electron spin along a chosen axis by appropriate basis rotations followed by Z-basis optical readout [14]. To mitigate potential dephasing of the nuclear spin induced by the electron-spin measurement, we make the electron-spin measurement nondestructive by using a short, weak laser pulse and conditioning progression of the sequence on the outcome $|0\rangle$, i.e., the detection of a photon $[16,42]$ (see Supplemental Material for all readout parameters [53]). Following appropriate basis rotations, we then use the electron spin to measure the nuclear spin in the $X$ basis [16]. In this measurement, the electron is read out in a single shot with average fidelity $0.945(2)$ [14]. We independently characterize the nuclear spin readout, which is then used to correct for readout infidelities in subsequent measurements [53]. In order to reconstruct the full electronnuclear state, we perform the sequence with and without an additional electron $\pi$ pulse before the first readout [53].

The reconstructed density matrix from quantum-state tomography is shown in Fig. 4(b). The prepared state $\rho$ exhibits a fidelity with the target Bell state of $\mathcal{F}_{\text {Bell }}=$ $\left\langle\psi_{\text {Bell }}|\rho| \psi_{\text {Bell }}\right\rangle=0.972(8)$. Based upon a simple depolarizing noise model, we estimate the two-qubit gate fidelity to be $\mathcal{F}_{\text {gate }}=0.991(9)$ [53]. Additional characterization measurements in combination with numerical simulations indicate that the remaining infidelity can be mostly attributed to electron-spin dephasing due to noise from the electronic hardware [53].

\section{A TEN-QUBIT SOLID-STATE SPIN REGISTER}

We now show how the combination of our DDrf gate with previously developed gates and control techniques $[16,37]$ enables high-fidelity control of a ten-qubit hybrid spin register associated with a single NV center. Our register is composed of the electron and ${ }^{14} \mathrm{~N}$ spins of the $\mathrm{NV}$ center, along with eight ${ }^{13} \mathrm{C}$ nuclear spins (Fig. 1). Our quantum register is connected via the central electron spin. To demonstrate this, we first show that all nuclear spins can be entangled with the electron spin by following the protocol shown in Fig. 4(a). For the case of the nitrogen spin, initialization is performed by a measurement-based scheme which heralds the preparation in a particular 


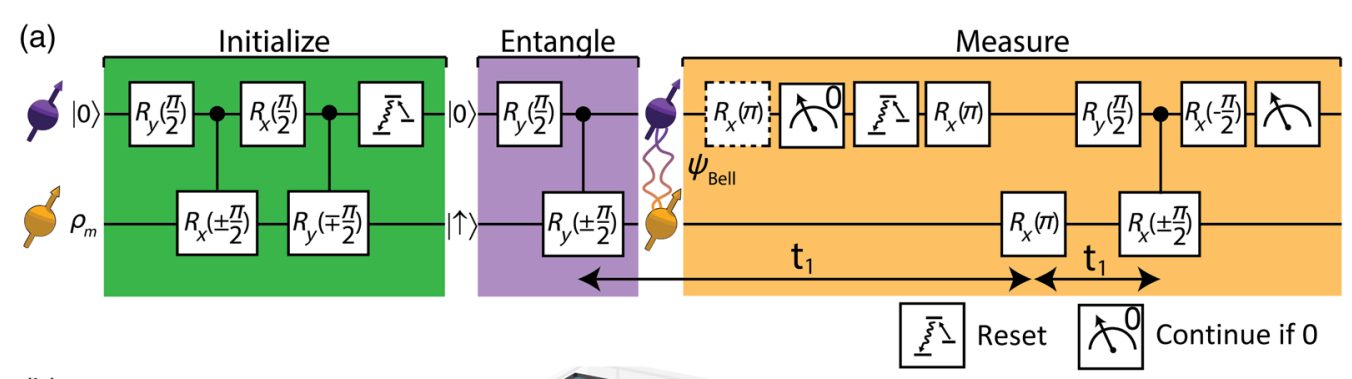

(b)

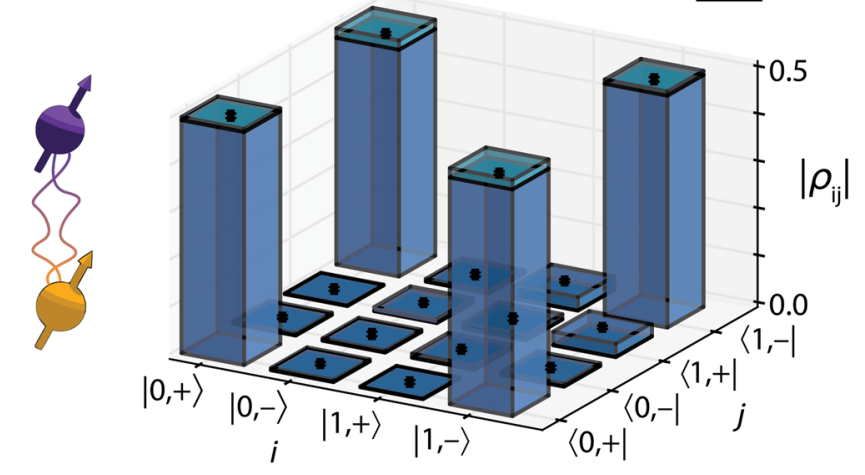

FIG. 4. (a) Experimental sequence to prepare an electron-nuclear Bell state and determine the expectation value of the two-qubit operator $Z X$. A series of single- and two-qubit gates are used to initialize the nuclear spin [16,37]. A subsequent $\pi / 2$ rotation and two-qubit gate generate the Bell state $\left|\psi_{\text {Bell }}\right\rangle=(|0+\rangle+|1-\rangle) / \sqrt{2}$. A measurement of the electron spin in the $Z$ basis is followed by an $X$-basis measurement of the nuclear spin through the electron spin. These measurements are separated by a nuclear spin echo, which is implemented to mitigate dephasing of the nuclear spin. The entire sequence is applied with and without an additional electron $\pi$ pulse (dashed box) before the first electron readout in order to reconstruct the electron state while ensuring that the measurement does not disturb the nuclear spin state $[16,42]$. (b) Density matrix of the electron-nuclear state after applying the sequence shown in (a) to qubit $\mathrm{C} 1$, reconstructed with state tomography. We correct for infidelities in the readout sequence characterized in separate measurements [53]. The DDrf gate parameters are $N=8, \tau=17 \tau_{L} \approx 39.4 \mu \mathrm{s}, \Omega / 2 \pi=1.09(3) \mathrm{kHz}$, and the total gate duration is $629 \mu \mathrm{s}$ compared with the nuclear spin $T_{2}^{*}=12.0(6) \mathrm{ms}$. We use error function pulse envelopes with a 7.5- $\mu$ s rise or fall time for each rf pulse to mitigate pulse distortions induced by the rf electronics [53]. The fidelity with the target Bell state is measured to be $\mathcal{F}_{\text {Bell }}=0.972(8)$. Lighter blue shading indicates the density matrix for the ideal state $\left|\psi_{\text {Bell }}\right\rangle$.

eigenstate. Compared to previous work [60], we realize an improved initialization fidelity $\left[\mathcal{F}_{\text {init }}=0.997(11)\right]$ by prepreparing the electron in the $m_{s}=-1$ state instead of a mixed state of $m_{s}=-1$ and +1 , and by repeating the measurement-based initialization sequence twice [53]. After initialization, we work in the $m_{I}=\{0,-1\}$ subspace and perform operations analogous to those for the ${ }^{13} \mathrm{C}$ nuclear spins, including the two-qubit gates using the DDrf scheme. Genuine entanglement is probed by measuring the nonzero matrix elements of the target state and confirmed by negativity of the entanglement witness $\mathcal{W}_{\text {Bell }}=\mathbb{1}-2\left|\psi_{\text {Bell }}\right\rangle\left\langle\psi_{\text {Bell }}\right|[61]$.

Next, we show that the register is fully connected by preparing entangled states for all possible pairs of spins. To prepare nuclear-nuclear entanglement, we implement a probabilistic measurement-based scheme [62], as shown in Fig. 5(a). We first prepare a three-qubit GHZ state comprising the electron and two nuclear spins $\left|\mathrm{GHZ}_{3}\right\rangle=$ $(|0++\rangle+|1--\rangle) / \sqrt{2}$ before performing a nondestructive $X$-basis measurement on the electron spin. The measurement ideally prepares the Bell state $\left|\Phi^{+}\right\rangle=(|++\rangle+$ $|--\rangle) / \sqrt{2}$ on the targeted pair of nuclear spins. Finally, we measure the necessary expectation values in order to reconstruct the nonzero matrix elements of this state and confirm bipartite entanglement [Fig. 5(b)].

The measured Bell state fidelities ranging from 0.63(3) to $0.97(1)$ are shown in Fig. 6. We attribute the variations in the measured values to differences in the two-qubit gate fidelities for each spin. In particular, the lower values measured for ${ }^{13} \mathrm{C}$ spins $\mathrm{C} 7$ and $\mathrm{C} 8$ are due to short coherence times in combination with long two-qubit gate durations necessitated by close spectral proximity to other spins [53]. All data are measured using a single set of gate parameters and using a single hardware configuration rather than separately optimizing for each pair of qubits.

\section{GENERATION OF $N$-QUBIT GHZ STATES}

Quantum-information processing tasks such as computations and error correction require the execution of complex algorithms comprising a large number of qubits. An important requirement for a quantum processor is thus the ability to perform operations on many of its constituents within a single algorithm. We test this capability by generating $N$-qubit GHZ-type states defined as 


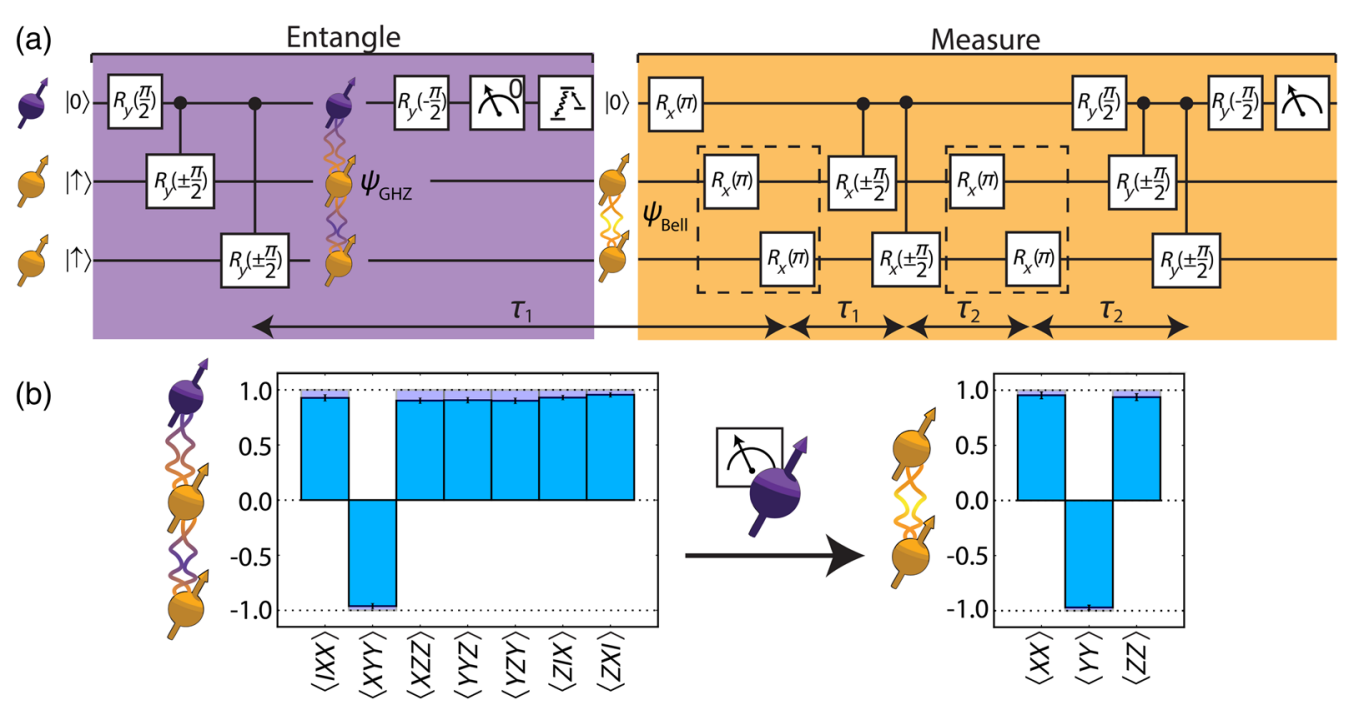

FIG. 5. (a) Experimental sequence for the preparation of a nuclear-nuclear Bell state and measurement of the two-qubit operator $Z Z$. After preparation of the electron-nuclear-nuclear $\mathrm{GHZ}$ state $\left|\mathrm{GHZ}_{3}\right\rangle=(|0++\rangle+|1--\rangle) / \sqrt{2}$, an $X$-basis measurement on the ancilla (electron spin) projects the nuclear spins into the Bell state $\left|\Phi^{+}\right\rangle=(|++\rangle+|--\rangle) / \sqrt{2}$. Measurement of the two-qubit correlations between the nuclear spins is then performed through the electron spin. Spin echoes (dashed boxes) built into the measurement sequence protect the nuclear spins from dephasing errors. (b) Measured expectation values (nonzero terms of the ideal state only) for the electronnuclear-nuclear state $\left|\mathrm{GHZ}_{3}\right\rangle$ and for the nuclear-nuclear state $\left|\Phi^{+}\right\rangle$. Blue (purple) bars show the experimental (ideal) expectation values for each operator. The nuclear-nuclear correlations are well preserved after a nondestructive measurement of the electron spin in the $X$ basis.

$$
\left|\mathrm{GHZ}_{N}\right\rangle=\frac{1}{\sqrt{2}}\left(|0\rangle \otimes|+\rangle^{\otimes(N-1)}+|1\rangle \otimes|-\rangle^{\otimes(N-1)}\right) .
$$

To generate such states, we follow the sequence shown in Fig. 7(a). First, $N-1$ nuclear spins are initialized in the

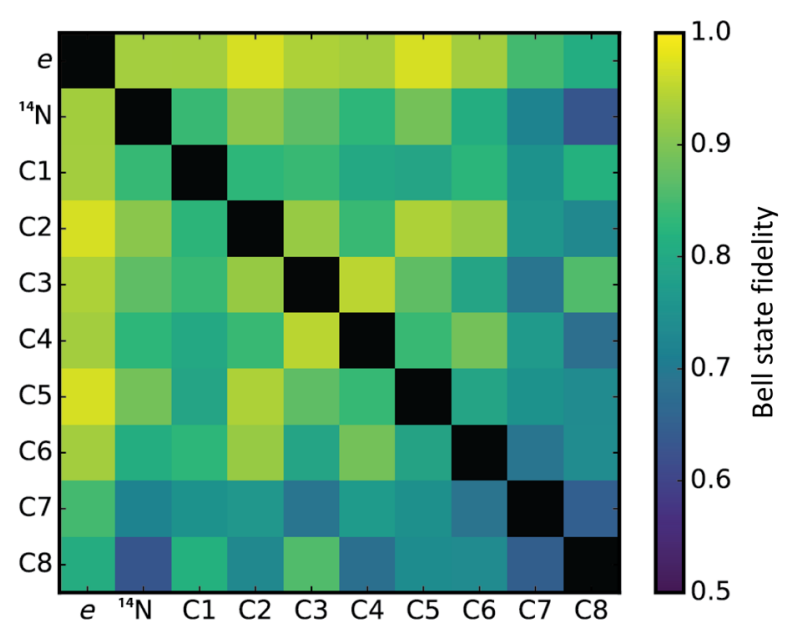

FIG. 6. Measured Bell state fidelities for all pairs of qubits in the ten-qubit register. Genuine entanglement is confirmed in all cases, as witnessed by a fidelity exceeding 0.5 with the target state. Qubits C1, C7, C8, and ${ }^{14} \mathrm{~N}$ are controlled using DDrf gates (Sec. II). Qubits C2, C3, C4, C5, and C6 are controlled using the methods described in Taminiau et al. [37], as their hyperfine interaction parameters enable high-fidelity control using previously optimized gates. state $|\uparrow\rangle$. Next, we prepare the electron spin in a superposition state and perform sequential controlled rotation gates between the electron and nuclear spins.

Characterizing the full quantum state for a system of this size is an expensive task due to the dimensionality of the associated Hilbert space. However, we can determine if the state exhibits genuine multipartite entanglement of all $N$ qubits using an entanglement witness with a reduced subset of measurement bases [61]. For a GHZ state with system size $N$, there exist $2^{N}$ operators from which the nonzero elements of the density matrix can be reconstructed by linear inversion and from which a fidelity with the target state can be calculated. Negativity of the entanglement witness $\mathcal{W}_{\mathrm{GHZ}}=\mathbb{1} / 2-\left|\mathrm{GHZ}_{N}\right\rangle\left\langle\mathrm{GHZ}_{N}\right|$ heralds genuine multipartite entanglement [61]. We determine the required expectation values of products of Pauli operators on the register via the electron spin. In these experiments, the readout sequence is modified slightly. Prior to the readout of the electron-spin state, we rotate the nitrogen spin such that the desired measurement basis is mapped to the $Z$ basis. This ensures that the population in the measurement basis is protected from dephasing during the optical readout of the electron spin, which is caused by the large electronnitrogen hyperfine coupling in the excited state $[53,63]$.

As the number of qubits is increased, a new challenge arises: The total sequence time becomes comparable to, or even exceeds the natural dephasing times $\left(T_{2}^{*}\right)$ of the nuclear spins. In order to preserve the nuclear spin coherence, we insert spin-echo pulses (rf $\pi$ pulses) into the 
(a)

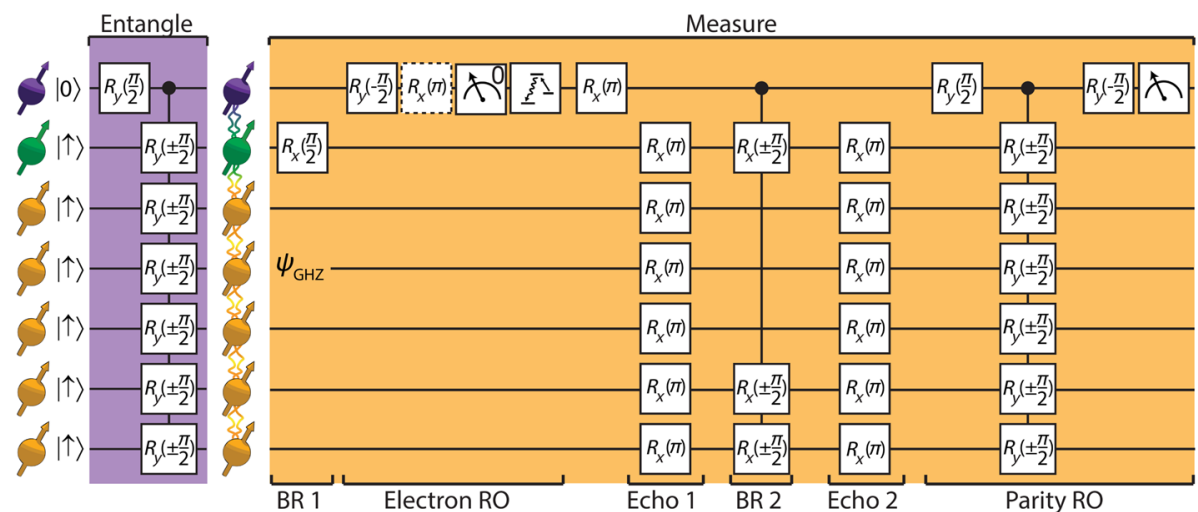

(b)

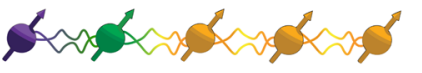

(d)

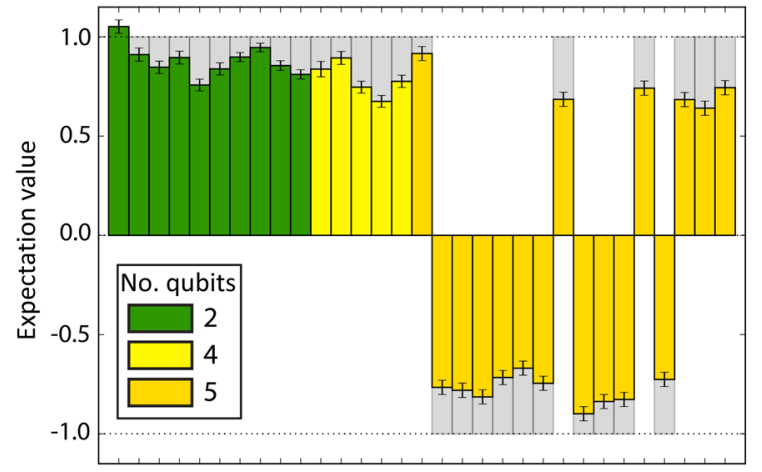

Multiqubit operators

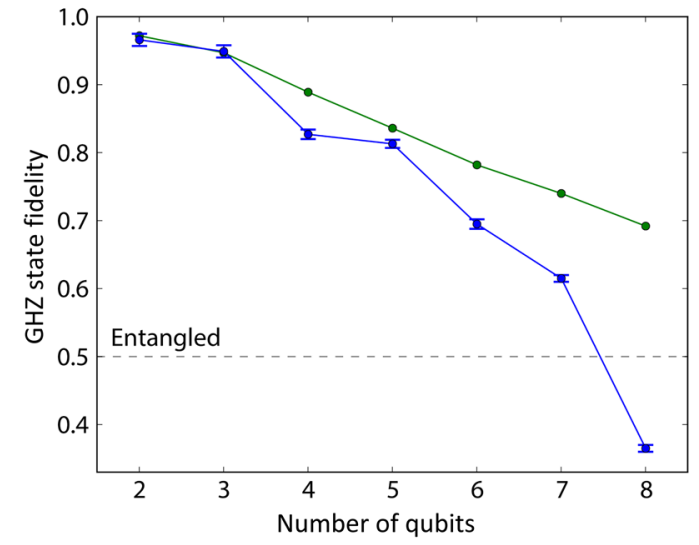

(c)
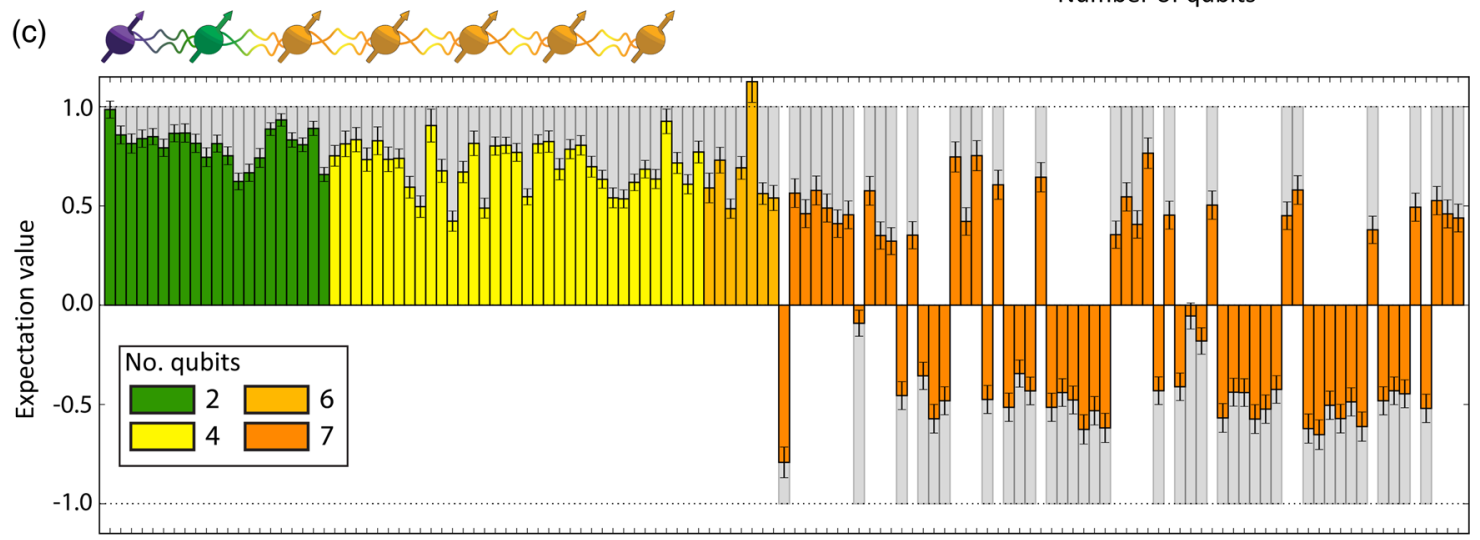

Multiqubit operators

FIG. 7. (a) Experimental sequence to prepare a seven-qubit GHZ state $\left|\mathrm{GHZ}_{7}\right\rangle$ (purple) and determine the expectation value of the seven-qubit operator $X Y Y Y Y Z Z$ (orange). The measurement sequence is broken down into basis rotations (BR 1,2), an electron readout (RO), nuclear spin echoes (echo 1,2), and a multiqubit readout of the nuclear spins. All operations are applied sequentially (in the same way as shown in Fig. 5), but some are shown in parallel for clarity. (b),(c) Bar plots showing the measured expectation values (nonzero terms of the ideal state only) after preparing the five-spin (b) and seven-spin (c) GHZ states. The colors indicate the number of qubits involved, i.e., the number of (nonidentity) operators in the expectation value (inset). Gray bars show the ideal expectation values. See the Supplemental Material [53] for the operator corresponding to each bar. The fidelity with the target state is 0.804(6) (b) and 0.589(5) (c), confirming genuine multipartite entanglement in both cases. (d) Plot of GHZ state fidelity against the number of constituent qubits. A value above 0.5 confirms genuine $N$-qubit entanglement. The blue points are the measured data, while the green points are theoretical predictions assuming a simple depolarizing noise model whose parameters are extracted from single- and two-qubit experiments. Numerical values are given in the Supplemental Material [53].

sequence to refocus each spin at the point of the next operation performed upon it. In the Supplemental Material [53], we derive a general solution that can be used to algorithmically construct echo sequences that avoid any overlap in gates and that minimize idle time with the electron spin in a superposition state. 
In Figs. 7(b) and 7(c), we show measurements for $N=5$ and $N=7$ qubits. In Fig. 7(d), we present the measured fidelities with the target GHZ states for two to eight qubits, along with theoretical values as predicted by a depolarizing-noise model based on the individual two-qubit gate fidelities [53]. The growing discrepancy between the measured and predicted values for larger $N$ suggests residual cross talk between the qubits, which is not taken into account in the model. For registers comprising up to seven spins, we observe negativity of the witness $\mathcal{W}_{\mathrm{GHZ}}$, revealing genuine $N$-qubit entanglement of up to seven qubits with high statistical significance.

\section{A LONG-LIVED QUANTUM MEMORY}

The nuclear spin qubits surrounding the NV center are promising candidates for quantum memories with long coherence times $[32,64]$. Here we investigate the coherence properties of the register under dynamical decoupling and show that an arbitrary single-qubit state can be protected for over 75 s. Furthermore, we show that two-qubit entanglement can be preserved beyond $10 \mathrm{~s}$.

We first investigate the coherence of individual nuclear spin qubits under dynamical decoupling. After initializing the nuclear spin in the state $|+\rangle$, we prepare the electron in the state $|1\rangle$ [electron $T_{1}=3.6(3) \times 10^{3} \mathrm{~s}$ [47] ]. This has two effects. First, it allows us to perform selective if $\pi$ pulses on the target nuclear spin. Second, the magnetic field gradient imposed by the electronnuclear hyperfine interaction induces a frozen core, which suppresses flip-flop interactions between nuclear spins $[65,66]$ and thereby reduces the noise the spins are exposed to.

The observed spin-echo coherence times $T_{2}^{\alpha=1}$, with $\alpha$ the number of rf pulses, vary between 0.26(3) and 0.77(4) s for the eight ${ }^{13} \mathrm{C}$ spins. For the ${ }^{14} \mathrm{~N}$ spin, we find $2.3(2) \mathrm{s}$, consistent with the smaller gyromagnetic ratio by a factor of 3.4. The range of coherence times observed for the ${ }^{13} \mathrm{C}$ spins is likely caused by differences in the microscopic environment of each spin. In particular, ${ }^{13} \mathrm{C}$ spins close to the NV center are in the heart of the frozen core and generally tend to couple predominantly to the part of the spin environment for which the dynamics are also suppressed most strongly. Spins farther from the NV tend to couple more strongly to the spin environment outside the frozen core. This explanation is consistent with the observation that the spin with the longest $T_{2}^{\alpha=1}$ of $0.77(4) \mathrm{s}$ is located closest [C1, $r=0.53(5) \mathrm{nm}$ [58]] to the vacancy lattice site, while the shortest $T_{2}^{\alpha=1}$ of $0.26(3) \mathrm{s}$ is found for a spin at a larger distance [C8, $r=1.04(4) \mathrm{nm}$ [58]]. As expected, increasing the number of decoupling pulses leads to an increase in the measured coherence times. For $\alpha=256$ pulses, the decay time of C5 reaches $T_{2}^{\alpha=256}=12.9(4) \mathrm{s}$, while for the ${ }^{14} \mathrm{~N}$ spin, we measure
$T_{2}^{\alpha=256}=63(2) \mathrm{s}$ (see Supplemental Material Fig. 2 [53]). For the other ${ }^{13} \mathrm{C}$ spins for which we measure $T_{2}^{\alpha=256}$, we find a range of values from 4(1) to 25(4) $\mathrm{s}$ [53].

To confirm that arbitrary quantum states can be protected, we prepare the six cardinal states and measure the average state fidelity under dynamical decoupling. The measured decay curves for spin $\mathrm{C} 5$ and the ${ }^{14} \mathrm{~N}$ spin are shown in Figs. 8(a) and 8(b), where $\alpha$ is varied from 1 to 256 . With 256 pulses, we measure a state fidelity exceeding the classical memory bound of $\frac{2}{3}$ at a time of $16.8 \mathrm{~s}$ for C5 and at a time of $75.3 \mathrm{~s}$ for the ${ }^{14} \mathrm{~N}$ spin.

The coherence times demonstrated here are the longest reported for individual qubits in the solid state and exceed values for isolated nuclear spin qubits in isotopically purified materials [24,32,33]. More importantly, however, in our register we realize these long coherence times while maintaining access to ten coupled spin qubits.

We exploit the multiqubit nature of the register to investigate the protection of entangled states of two ${ }^{13} \mathrm{C}$ spin qubits. After preparing the state $\left|\Phi^{+}\right\rangle=(|++\rangle+$ $|--\rangle) / \sqrt{2}$ following the sequence shown in Fig. 5(a), we again prepare the electron in the state $|1\rangle$. We then measure the Bell state fidelity as a function of total evolution time for $\alpha=1$ to $\alpha=256$ pulses. Note that since $\left|\Phi^{+}\right\rangle$is an eigenstate of $Z Z$, its evolution is not affected by the coupling between the two qubits, which is predominantly dipolar and of the form $C I_{z} I_{z}$, with $C=1.32(4) \mathrm{Hz}$ [58]. The measured fidelities are plotted in Fig. 8(c). For $\alpha=256$ decoupling pulses, we confirm the preservation of entanglement for $>10 \mathrm{~s}$, as quantified by a fidelity exceeding 0.5 with the desired Bell state.

With the capability to protect multiqubit quantum states, it becomes important to consider additional effects that may affect their coherence, such as the presence of correlated noise. As a first experimental step towards understanding such effects, we use entangled states of nuclear spins to explore spatial correlations within the noise environment. We perform experiments on two pairs of ${ }^{13} \mathrm{C}$ spins. We prepare two Bell states for each pair, one exhibiting even $Z Z$ parity, which, written in the $Z$ basis, is given by $\left|\Phi^{+}\right\rangle=(|\downarrow \downarrow\rangle+|\uparrow \uparrow\rangle) / \sqrt{2}$, and another exhibiting odd $Z Z$ parity, $\left|\Psi^{-}\right\rangle=(|\downarrow \uparrow\rangle-|\uparrow \downarrow\rangle) / \sqrt{2}$. The difference in the coherence times of these two states gives an indication of the amount of correlation in the noise experienced by the two spins [67]. In the case of perfectly correlated noise, one would expect the state $\left|\Phi^{+}\right\rangle$to decay at four times the single-qubit decay rate (superdecoherence), while the state $\left|\Psi^{-}\right\rangle$would form a decoherence-free subspace $[68,69]$. In contrast, for completely uncorrelated noise, the coherence times for the two states would be identical.

We measure the coherence times for the two Bell states, varying the total evolution time for the case of a single spinecho pulse $(\alpha=1)$ with the electron spin prepared in the state $|1\rangle$. In Fig. 8(d), we plot the normalized coherence 
(a)
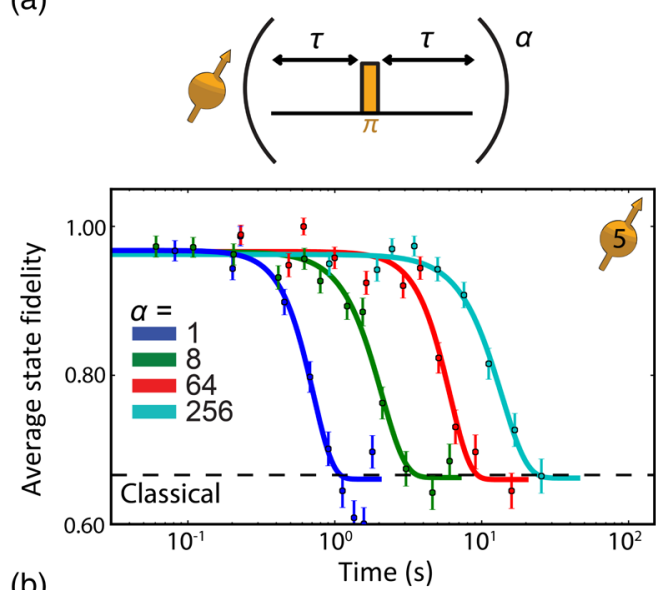

(b)

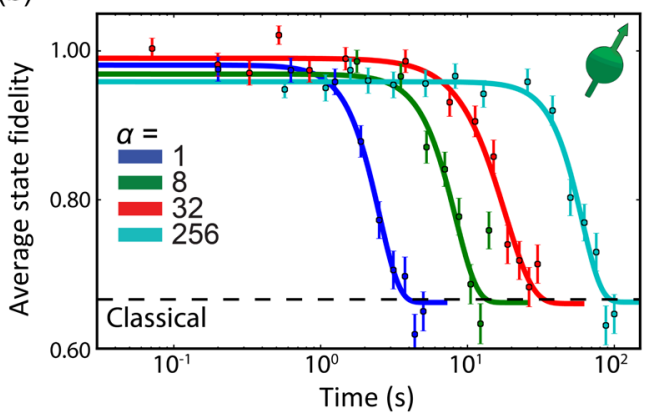

(c)
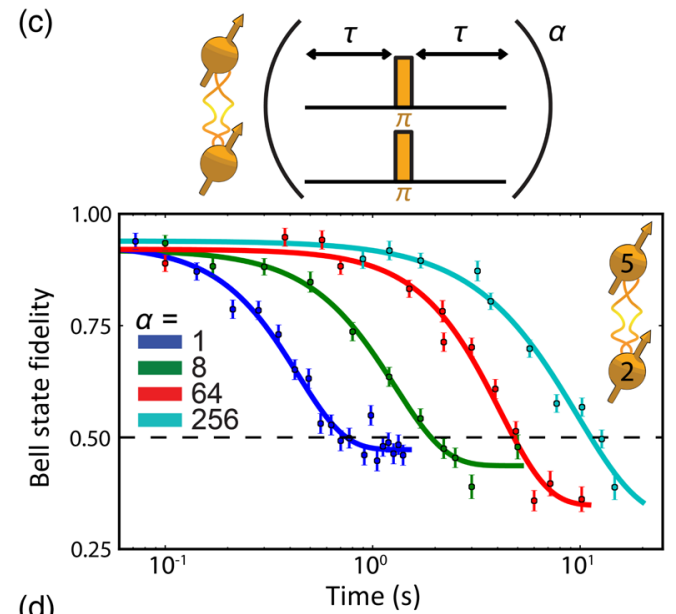

(d)

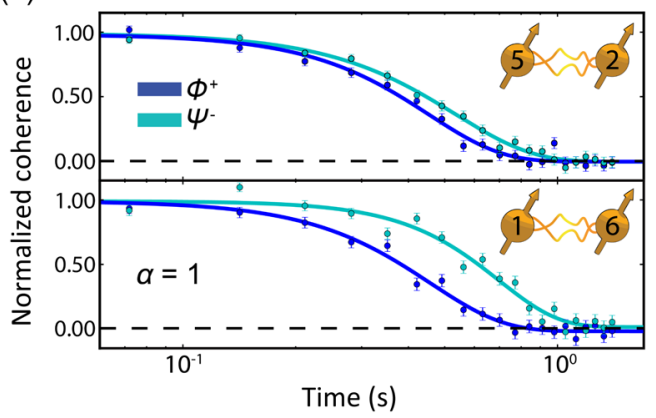

FIG. 8. (a) Dynamical decoupling for spin C5. With $\alpha=256$ pulses, the average state fidelity of the six cardinal states is measured to be $0.73(2)$ after $16.8 \mathrm{~s}$, above the limit of $\frac{2}{3}$ for a classical memory with a confidence of $99.7 \%$ (upper-tailed $Z$ test). Solid lines are fit to the function $f(t)=A+B e^{-(t / T)^{n}}$. The offset $A$ is fixed using the average fidelity of the input states $|\uparrow\rangle$ and $|\downarrow\rangle$, which show no decay on these timescales. $B, T$, and $n$ are fit parameters which account for the decay of the fidelity due to interactions with the nuclear spin bath, external noise, and pulse errors. (b) Dynamical decoupling of the ${ }^{14} \mathrm{~N}$ spin. For $\alpha=256$ pulses, the average state fidelity at $75.3 \mathrm{~s}$ is 0.73 (3), which is above the bound for a classical memory with $99.4 \%$ confidence. (c) Dynamical decoupling of a pair of ${ }^{13} \mathrm{C}$ spins prepared in the Bell state $\left|\Phi^{+}\right\rangle$. Solid lines are fits to $f(t)$, but with $A$ as a free parameter to account for the observed decrease in the $Z Z$ correlations at large pulse numbers, likely due to pulse errors. With 256 decoupling pulses, genuine two-qubit entanglement is witnessed at times up to $10.2 \mathrm{~s}$, where we observe a fidelity of $0.57(2)$ with the target Bell state ( $99.9 \%$ confidence of entanglement). In addition, interpolation of the fit yields 11.3(8) s as the point where the fidelity crosses 0.5 [53]. (d) Normalized coherence $(\langle X X\rangle \pm\langle Y Y\rangle) / 2 \mathcal{N}$, where $\mathcal{N}$ is a normalization factor, for two pairs of ${ }^{13} \mathrm{C}$ spins prepared in both the even- and odd-parity Bell states $\left|\Phi^{+}\right\rangle=(|\downarrow \downarrow\rangle+|\uparrow \uparrow\rangle) / \sqrt{2}$ and $\left|\Psi^{-}\right\rangle=(|\downarrow \uparrow\rangle-|\downarrow \uparrow\rangle) / \sqrt{2}$. Solid lines are fits to $f(t)$ with $A=0$ and $B=1$. For pair 1, the fitted decay times $T$ are $0.45(2)$ and $0.54(1)$ $\mathrm{s}$ for the states $\left|\Phi^{+}\right\rangle$and $\left|\Psi^{-}\right\rangle$, respectively. For pair 2, the equivalent values are 0.46(2) and 0.70(3) s.

signal for both Bell states and for both pairs of qubits. A statistically significant difference between the decay curves for the two Bell states is found for both pairs, where the odd-parity state $\left|\Psi^{-}\right\rangle$decays more slowly than the evenparity state $\left|\Phi^{+}\right\rangle$, indicating partly correlated noise in the system. We can relate the size of the effect to the distance between the spins in the pairs, which has been characterized in separate work [58]. This reveals that the pair with a smaller separation [C1 and C6, distance 0.96(3) nm] shows more correlation than the pair with a larger separation [C5 and $\mathrm{C} 2$, $1.38(7) \mathrm{nm}$ ]. This observation is consistent with the idea that spatially close spins tend to couple to the same nuclear spin environment, and therefore experience correlated noise, although large deviations from this rule are expected to be possible for specific cases [67]. Characterizing such correlated noise provides new opportunities to investigate the physics of decoherence in spin baths [67] and to develop and test quantum-error-correction schemes that are tailored for specific correlated noise [70,71].

\section{CONCLUSION}

In conclusion, we develop a novel electron-nuclear twoqubit gate and apply these gates to realize a ten-qubit solidstate spin register that can protect an arbitrary single-qubit state for over one minute. The techniques developed in this work can be readily implemented for multiqubit control in a variety of other donor and defect platforms, including spin$1 / 2[7,9,13,20,28,54,55]$ and contact hyperfine [21,34] systems, for which many previous gate designs are challenging to apply [37,48-50]. Further improvements in selectivity and fidelity of the gates are anticipated to be 
possible by (optimal) shaping of the rf pulses [38,56,57] and by reducing electronic noise. Additionally, the use of direct $\mathrm{rf}$ driving opens the possibility to perform gates in parallel on multiple qubits. Combined with already demonstrated long-range optical entanglement [29-31], our multiqubit register paves the way for the realization of rudimentary few-node quantum networks comprising tens of qubits. This will enable the investigation of basic errorcorrection codes and algorithms over quantum networks [44-46]. Finally, looking beyond quantum information, the gate sequences developed here also enable new quantumsensing methods [58].

\section{ACKNOWLEDGMENTS}

We thank T. van der Sar for valuable discussions and preliminary experiments. We thank V.V. Dobrovitski and R. Hanson for valuable discussions, R.F.L. Vermeulen and R. N. Schouten for assistance with the rf electronics, and M. Eschen for assistance with the experimental setup. This work was supported by the Netherlands Organisation for Scientific Research (NWO/OCW), as part of the Frontiers of Nanoscience (NanoFront) program, through a Vidi grant, and as part of the Quantum Software Consortium programme (Project No. 024.003.037/3368).

[1] D. D. Awschalom, R. Hanson, J. Wrachtrup, and B. B. Zhou, Quantum Technologies with Optically Interfaced Solid-State Spins, Nat. Photonics 12, 516 (2018).

[2] F. A. Zwanenburg, A. S. Dzurak, A. Morello, M. Y. Simmons, L. C. L. Hollenberg, G. Klimeck, S. Rogge, S. N. Coppersmith, and M. A. Eriksson, Silicon Quantum Electronics, Rev. Mod. Phys. 85, 961 (2013).

[3] G. De Lange, Z. H. Wang, D. Riste, V. V. Dobrovitski, and R. Hanson, Universal Dynamical Decoupling of a Single Solid-State Spin from a Spin Bath, Science 330, 60 (2010).

[4] G. D. Fuchs, V. V. Dobrovitski, D. M. Toyli, F. J. Heremans, and D. D. Awschalom, Gigahertz Dynamics of a Strongly Driven Single Quantum Spin, Science 326, 1520 (2009).

[5] D. J. Christle, A. L. Falk, P. Andrich, P. V. Klimov, J. U. Hassan, N. T. Son, E. Janzén, T. Ohshima, and D. D. Awschalom, Isolated Electron Spins in Silicon Carbide with Millisecond Coherence Times, Nat. Mater. 14, 160 (2015).

[6] H. Seo, A. L. Falk, P. V. Klimov, K. C. Miao, G. Galli, and D. D. Awschalom, Quantum Decoherence Dynamics of Divacancy Spins in Silicon Carbide, Nat. Commun. 7, 12935 (2016).

[7] D. D. Sukachev, A. Sipahigil, C. T. Nguyen, M. K. Bhaskar, R. E. Evans, F. Jelezko, and M. D. Lukin, Silicon-Vacancy Spin Qubit in Diamond: A Quantum Memory Exceeding 10 ms with Single-Shot State Readout, Phys. Rev. Lett. 119, 223602 (2017).

[8] T. Iwasaki, F. Ishibashi, Y. Miyamoto, Y. Doi, S. Kobayashi, T. Miyazaki, K. Tahara, K. D. Jahnke, L. J. Rogers, B. Naydenov et al., Germanium-Vacancy Single Color Centers in Diamond, Sci. Rep. 5, 12882 (2015).
[9] P. Siyushev, M. H. Metsch, A. Ijaz, J. M. Binder, M. K. Bhaskar, D. D. Sukachev, A. Sipahigil, R. E. Evans, C. T. Nguyen, M. D. Lukin et al., Optical and Microwave Control of Germanium-Vacancy Center Spins in Diamond, Phys. Rev. B 96, 081201(R) (2017).

[10] J. N. Becker, B. Pingault, D. Groß, M. Gündoğan, N. Kukharchyk, M. Markham, A. Edmonds, M. Atatüre, P. Bushev, and C. Becher, All-Optical Control of the SiliconVacancy Spin in Diamond at Millikelvin Temperatures, Phys. Rev. Lett. 120, 053603 (2018).

[11] B. Pingault, D.-D. Jarausch, C. Hepp, L. Klintberg, J. N. Becker, M. Markham, C. Becher, and M. Atatüre, Coherent Control of the Silicon-Vacancy Spin in Diamond, Nat. Commun. 8, 15579 (2017).

[12] M. E. Trusheim, B. Pingault, N. H. Wan, L. De Santis, K. C. Chen, M. Walsh, J. J. Rose, J. N. Becker, E. Bersin, G. Malladi et al., Transform-Limited Photons from a Tin-Vacancy Spin in Diamond, arXiv:1811 .07777 .

[13] A. E. Rugar, C. Dory, S. Sun, and J. Vučković, Characterization of Optical and Spin Properties of Single TinVacancy Centers in Diamond Nanopillars, Phys. Rev. B 99, 205417 (2019).

[14] L. Robledo, L. Childress, H. Bernien, B. Hensen, P. F. A. Alkemade, and R. Hanson, High-Fidelity Projective ReadOut of a Solid-State Spin Quantum Register, Nature (London) 477, 574 (2011).

[15] J. J. Pla, K. Y. Tan, J. P. Dehollain, W. H. Lim, J. J. L. Morton, F. A. Zwanenburg, D. N. Jamieson, A. S. Dzurak, and A. Morello, High-Fidelity Readout and Control of a Nuclear Spin Qubit in Silicon, Nature (London) 496, 334 (2013).

[16] J. Cramer, N. Kalb, M. A. Rol, B. Hensen, M. S. Blok, M. Markham, D. J. Twitchen, R. Hanson, and T. H. Taminiau, Repeated Quantum Error Correction on a Continuously Encoded Qubit by Real-Time Feedback, Nat. Commun. 7, 11526 (2016).

[17] G. Waldherr, Y. Wang, S. Zaiser, M. Jamali, T. SchulteHerbruggen, H. Abe, T. Ohshima, J. Isoya, J. F. Du, P. Neumann et al., Quantum Error Correction in a Solid-State Hybrid Spin Register, Nature (London) 506, 204 (2014).

[18] G. Wolfowicz, P.-A. Mortemousque, R. Guichard, S. Simmons, M. L. W. Thewalt, K. M. Itoh, and J. J. L. Morton, ${ }^{29} \mathrm{Si}$ Nuclear Spins as a Resource for Donor Spin Qubits in Silicon, New J. Phys. 18, 023021 (2016).

[19] G. Tosi, F. A. Mohiyaddin, V. Schmitt, S. Tenberg, R. Rahman, G. Klimeck, and A. Morello, Silicon Quantum Processor with Robust Long-Distance Qubit Couplings, Nat. Commun. 8, 450 (2017).

[20] M. H. Metsch, K. Senkalla, B. Tratzmiller, J. Scheuer, M. Kern, J. Achard, A. Tallaire, M. B. Plenio, P. Siyushev, and F. Jelezko, Initialization and Readout of Nuclear Spins via a Negatively Charged Silicon-Vacancy Center in Diamond, Phys. Rev. Lett. 122, 190503 (2019).

[21] B. Hensen, W. Huang, C.-H. Yang, K. W. Chan, J. Yoneda, T. Tanttu, F. E. Hudson, A. Laucht, K. M. Itoh, A. Morello, and A. S. Dzurak, A Silicon Quantum-Dot-Coupled Nuclear Spin Qubit, arXiv:1904.08260.

[22] F. Dolde, I. Jakobi, B. Naydenov, N. Zhao, S. Pezzagna, C. Trautmann, J. Meijer, P. Neumann, F. Jelezko, and 
J. Wrachtrup, Room-Temperature Entanglement between Single Defect Spins in Diamond, Nat. Phys. 9, 139 (2013).

[23] T. Yamamoto, C. Müller, L. P. McGuinness, T. Teraji, B. Naydenov, S. Onoda, T. Ohshima, J. Wrachtrup, F. Jelezko, and J. Isoya, Strongly Coupled Diamond Spin Qubits by Molecular Nitrogen Implantation, Phys. Rev. B 88, 201201 (R) (2013).

[24] S. Yang, Y. Wang, D. D. B. Rao, T. H. Tran, A. S. Momenzadeh, M. Markham, D. J. Twitchen, P. Wang, W. Yang, R. Stöhr et al., High-Fidelity Transfer and Storage of Photon States in a Single Nuclear Spin, Nat. Photonics 10, 507 (2016).

[25] E. Togan, Y. Chu, A. S. Trifonov, L. Jiang, J. Maze, L. Childress, M. V. G. Dutt, A. S. Sørensen, P. R. Hemmer, A.S. Zibrov et al., Quantum Entanglement between an Optical Photon and a Solid-State Spin Qubit, Nature (London) 466, 730 (2010).

[26] D. J. Christle, P. V. Klimov, F. Charles, K. Szász, V. Ivády, V. Jokubavicius, J. U. Hassan, M. Syväjärvi, W. F. Koehl, T. Ohshima et al., Isolated Spin Qubits in SiC with a HighFidelity Infrared Spin-to-Photon interface, Phys. Rev. X 7, 021046 (2017).

[27] A. Sipahigil, R. E. Evans, D. D. Sukachev, M. J. Burek, J. Borregaard, M. K. Bhaskar, C. T. Nguyen, J. L. Pacheco, H. A. Atikian, C. Meuwly et al., An Integrated Diamond Nanophotonics Platform for Quantum-Optical Networks, Science 354, 847 (2016).

[28] R. E. Evans, M. K. Bhaskar, D. D. Sukachev, C. T. Nguyen, A. Sipahigil, M. J. Burek, B. Machielse, G. H. Zhang, A. S. Zibrov, E. Bielejec et al., Photon-Mediated Interactions between Quantum Emitters in a Diamond Nanocavity, Science 362, 662 (2018).

[29] H. Bernien, B. Hensen, W. Pfaff, G. Koolstra, M. S. Blok, L. Robledo, T. H. Taminiau, M. Markham, D. J. Twitchen, L. Childress et al., Heralded Entanglement between SolidState Qubits Separated by Three Metres, Nature (London) 497, 86 (2013).

[30] B. Hensen, H. Bernien, A. E. Dréau, A. Reiserer, N. Kalb, M. S. Blok, J. Ruitenberg, R. F. L. Vermeulen, R. N. Schouten, C. Abellán et al., Loophole-Free Bell Inequality Violation Using Electron Spins Separated by 1.3 Kilometres, Nature (London) 526, 682 (2015).

[31] P. C. Humphreys, N. Kalb, J. P. J. Morits, R. N. Schouten, R. F. L. Vermeulen, D. J. Twitchen, M. Markham, and R. Hanson, Deterministic Delivery of Remote Entanglement on a Quantum Network, Nature (London) 558, 268 (2018).

[32] P. C. Maurer, G. Kucsko, C. Latta, L. Jiang, N. Y. Yao, S. D. Bennett, F. Pastawski, D. Hunger, N. Chisholm, M. Markham et al., Room-Temperature Quantum Bit Memory Exceeding One Second, Science 336, 1283 (2012).

[33] J. T. Muhonen, J. P. Dehollain, A. Laucht, F. E. Hudson, R. Kalra, T. Sekiguchi, K. M. Itoh, D. N. Jamieson, J. C. McCallum, A. S. Dzurak et al., Storing Quantum Information for 30 Seconds in a Nanoelectronic Device, Nat. Nanotechnol. 9, 986 (2014).

[34] J. P. Dehollain, S. Simmons, J. T. Muhonen, R. Kalra, A. Laucht, F. Hudson, K. M. Itoh, D. N. Jamieson, J. C. McCallum, A. S. Dzurak et al., Bell's Inequality Violation with Spins in Silicon, Nat. Nanotechnol. 11, 242 (2016).
[35] S. Freer, S. Simmons, A. Laucht, J. T. Muhonen, J. P. Dehollain, R. Kalra, F. A. Mohiyaddin, F. E. Hudson, K. M. Itoh, J. C. McCallum et al., A Single-Atom Quantum Memory in Silicon, Quantum Sci. Technol. 2, 015009 (2017).

[36] T. van der Sar, Z. H. Wang, M. S. Blok, H. Bernien, T. H. Taminiau, D. M. Toyli, D. A. Lidar, D. D. Awschalom, R. Hanson, and V. V. Dobrovitski, Decoherence-Protected Quantum Gates for a Hybrid Solid-State Spin Register, Nature (London) 484, 82 (2012).

[37] T. H. Taminiau, J. Cramer, T. van der Sar, V. V. Dobrovitski, and R. Hanson, Universal Control and Error Correction in Multi-Qubit Spin Registers in Diamond, Nat. Nanotechnol. 9, 171 (2014).

[38] X. Rong, J. Geng, F. Shi, Y. Liu, K. Xu, W. Ma, F. Kong, Z. Jiang, Y. Wu, and J. Du, Experimental Fault-Tolerant Universal Quantum Gates with Solid-State Spins under Ambient Conditions, Nat. Commun. 6, 8748 (2015).

[39] S. Zaiser, T. Rendler, I. Jakobi, T. Wolf, S.-Y. Lee, S. Wagner, V. Bergholm, T. Schulte-Herbrüggen, P. Neumann, and J. Wrachtrup, Enhancing Quantum Sensing Sensitivity by a Quantum Memory, Nat. Commun. 7, 12279 (2016).

[40] T. Unden, D. Louzon, M. Zwolak, W. Zurek, and F. Jelezko, Revealing the Emergence of Classicality in NitrogenVacancy Centers, arXiv:1809.10456.

[41] Y.-Y. Huang, Y.-K. Wu, F. Wang, P.-Y. Hou, W.-B. Wang, W.-G. Zhang, W.-Q. Lian, Y.-Q. Liu, H.-Y. Wang, H.-Y. Zhang et al., Experimental Realization of Robust Geometric Quantum Gates with Solid-State Spins, Phys. Rev. Lett. 122, 010503 (2019).

[42] N. Kalb, A. A. Reiserer, P. C. Humphreys, J. J. W. Bakermans, S. J. Kamerling, N. H. Nickerson, S. C. Benjamin, D. J. Twitchen, M. Markham, and R. Hanson, Entanglement Distillation between Solid-State Quantum Network Nodes, Science 356, 928 (2017).

[43] S. B. van Dam, J. Cramer, T. H. Taminiau, and R. Hanson, Multipartite Entanglement Generation and Contextuality Tests Using Non-Destructive Three-Qubit Parity Measurements, Phys. Rev. Lett. 123, 050401 (2019).

[44] B. M. Terhal, Quantum Error Correction for Quantum Memories, Rev. Mod. Phys. 87, 307 (2015).

[45] N. H. Nickerson, Y. Li, and S. C. Benjamin, Topological Quantum Computing with a Very Noisy Network and Local Error Rates Approaching One Percent, Nat. Commun. 4, 1756 (2013).

[46] N. H. Nickerson, J. F. Fitzsimons, and S.C. Benjamin, Freely Scalable Quantum Technologies Using Cells of 5-to-50 Qubits with Very Lossy and Noisy Photonic Links, Phys. Rev. X 4, 041041 (2014).

[47] M. H. Abobeih, J. Cramer, M. A. Bakker, N. Kalb, M. Markham, D. J. Twitchen, and T. H. Taminiau, One-Second Coherence for a Single Electron Spin Coupled to a MultiQubit Nuclear-Spin Environment, Nat. Commun. 9, 2552 (2018).

[48] S. Kolkowitz, Q. P. Unterreithmeier, S. D. Bennett, and M. D. Lukin, Sensing Distant Nuclear Spins with a Single Electron Spin, Phys. Rev. Lett. 109, 137601 (2012).

[49] T. H. Taminiau, J. J. T. Wagenaar, T. van der Sar, F. Jelezko, V. V. Dobrovitski, and R. Hanson, Detection and Control of 
Individual Nuclear Spins Using a Weakly Coupled Electron Spin, Phys. Rev. Lett. 109, 137602 (2012).

[50] N. Zhao, J. Honert, B. Schmid, M. Klas, J. Isoya, M. Markham, D. Twitchen, F. Jelezko, R.-B. Liu, H. Fedder, and J. Wrachtrup, Sensing Single Remote Nuclear Spins, Nat. Nanotechnol. 7, 657 (2012).

[51] Z.-Yu Wang, J. Casanova, and M. B. Plenio, Delayed Entanglement Echo for Individual Control of a Large Number of Nuclear Spins, Nat. Commun. 8, 14660 (2017).

[52] M. Pfender, N. Aslam, H. Sumiya, S. Onoda, P. Neumann, J. Isoya, C. A. Meriles, and J. Wrachtrup, Nonvolatile Nuclear Spin Memory Enables Sensor-Unlimited Nanoscale Spectroscopy of Small Spin Clusters, Nat. Commun. 8, 834 (2017).

[53] See Supplemental Material at http://link.aps.org/ supplemental/10.1103/PhysRevX.9.031045, which contains experimental details and parameters, all measured coherence times and fidelities, modeling of the expected fidelities, and the generalized theoretical description of the gate dynamics.

[54] T. Müller, C. Hepp, B. Pingault, E. Neu, S. Gsell, M. Schreck, H. Sternschulte, D. Steinmüller-Nethl, C. Becher, and M. Atatüre, Optical Signatures of Silicon-Vacancy Spins in Diamond, Nat. Commun. 5, 3328 (2014).

[55] G. Thiering and A. Gali, Ab Initio Magneto-Optical Spectrum of Group-IV Vacancy Color Centers in Diamond, Phys. Rev. X 8, 021063 (2018).

[56] L. M. K. Vandersypen and I. L. Chuang, NMR Techniques for Quantum Control and Computation, Rev. Mod. Phys. 76, 1037 (2005).

[57] F. Dolde, V. Bergholm, Y. Wang, I. Jakobi, B. Naydenov, S. Pezzagna, J. Meijer, F. Jelezko, P. Neumann, T. SchulteHerbrüggen et al., High-Fidelity Spin Entanglement Using Optimal Control, Nat. Commun. 5, 3371 (2014).

[58] M. H. Abobeih, J. Randall, C. E. Bradley, H. P. Bartling, M. A. Bakker, M. J. Degen, M. Markham, D. J. Twitchen, and T. H. Taminiau, Atomic-Scale Imaging of a 27-NuclearSpin Cluster with a Single-Spin Quantum Sensor, arXiv: 1905.02095.

[59] L. Childress, M. V. G. Dutt, J. M. Taylor, A. S. Zibrov, F. Jelezko, J. Wrachtrup, P. R. Hemmer, and M. D. Lukin, Coherent Dynamics of Coupled Electron and Nuclear Spin Qubits in Diamond, Science 314, 281 (2006).
[60] N. Kalb, J. Cramer, D. J. Twitchen, M. Markham, R. Hanson, and T.H. Taminiau, Experimental Creation of Quantum Zeno Subspaces by Repeated Multi-Spin Projections in Diamond, Nat. Commun. 7, 13111 (2016).

[61] O. Gühne and G. Tóth, Entanglement Detection, Phys. Rep. 474, 1 (2009).

[62] W. Pfaff, T.H. Taminiau, L. Robledo, H. Bernien, M. Markham, D. J. Twitchen, and R. Hanson, Demonstration of Entanglement-by-Measurement of Solid-State Qubits, Nat. Phys. 9, 29 (2013).

[63] M. S. Blok, C. Bonato, M. L. Markham, D. J. Twitchen, V. V. Dobrovitski, and R. Hanson, Manipulating a Qubit through the Backaction of Sequential Partial Measurements and Real-Time Feedback, Nat. Phys. 10, 189 (2014).

[64] N. Kalb, P. C. Humphreys, J. J. Slim, and R. Hanson, Dephasing Mechanisms of Diamond-Based Nuclear-Spin Memories for Quantum Networks, Phys. Rev. A 97, 062330 (2018).

[65] G. R. Khutsishvili, Spin Diffusion and Magnetic Relaxation of Nuclei, Sov. Phys. JETP 15 (1962).

[66] R. Guichard, S. J. Balian, G. Wolfowicz, P. A. Mortemousque, and T. S. Monteiro, Decoherence of Nuclear Spins in the Frozen Core of an Electron Spin, Phys. Rev. B 91, 214303 (2015).

[67] D. Kwiatkowski and Ł. Cywiński, Decoherence of Two Entangled Spin Qubits Coupled to an Interacting Sparse Nuclear Spin Bath: Application to Nitrogen Vacancy Centers, Phys. Rev. B 98, 155202 (2018).

[68] D. A. Lidar, I. L. Chuang, and K. B. Whaley, DecoherenceFree Subspaces for Quantum Computation, Phys. Rev. Lett. 81, 2594 (1998).

[69] A. Reiserer, N. Kalb, M. S. Blok, K. J. M. van Bemmelen, T. H. Taminiau, R. Hanson, D. J. Twitchen, and M. Markham, Robust Quantum-Network Memory Using Decoherence-Protected Subspaces of Nuclear Spins, Phys. Rev. X 6, 021040 (2016).

[70] T. Monz, P. Schindler, J. T. Barreiro, M. Chwalla, D. Nigg, W. A. Coish, M. Harlander, W. Hänsel, M. Hennrich, and R. Blatt, 14-Qubit Entanglement: Creation and Coherence, Phys. Rev. Lett. 106, 130506 (2011).

[71] D. Layden, M. Chen, and P. Cappellaro, Efficient Quantum Error Correction of Dephasing Induced by a Common Fluctuator, arXiv:1903.01046. 